\title{
On the Kinematic Origin of the Luminosity-Pulse Lag Relationship in Gamma-ray Bursts
}

\author{
Jay D. Salmonson \\ Lawrence Livermore National Laboratory, Livermore, CA 94550
}

\begin{abstract}
This paper presents an interpretation based on gamma-ray burst source kinematics for the relationship found by Norris et al. between peak luminosity and energy-dependent pulse lag. I argue that the correlation should instead be between number luminosity and pulse lag. This interpretation improves the least-squares fit of this correlation for the known bursts by 25 percent or more. It also suggests a distance estimation scheme. I propose that this relationship is due to the variation in line-of-sight velocity among bursts. This interpretation allows one to speculate on the range of gamma-ray burst expansion velocities or the size of their jet opening angles.
\end{abstract}

Subject headings: gamma rays: bursts - gamma rays: theory

\section{Introduction}

It has recently been reported by Norris et al. $(2000 \mathrm{a}, \mathrm{b})$ that there is a relationship between the peak luminosity of gamma-ray bursts (GRB) and the pulse time lag between BATSE energy channels. Specifically, they find that the peak luminosity $L_{p k}$ is anticorrelated with the duration of the lag, $\Delta t$, according to $L_{p k} \approx 1.3 \times$ $(\Delta t / 0.01 \mathrm{sec})^{-1.15} \times 10^{53} \mathrm{ergs} / \mathrm{sec}$. This intriguing relationship is useful because it probes the properties of an ensemble of bursts and thereby can provide clues of the global dynamics of GRBs. It also has the potential for distance estimation to GRBs.

Several workers have attempted to explain the origin of the pulse lag, or so called pulse paradigm (Norris et al. 1996). Daigne \& Mochkovitch (1998) were able to produce a pulse lag by modeling the burst as a series of internal shocks, but obtained timescales an order of magnitude too large. However, as $\mathrm{Wu} \&$ Fenimore (2000) point out, the lag timescale is much longer than the synchrotron cooling time. Panaitescu \& Mészáros (1998) modeled the kinematics of internal shocks and found no evidence of pulse peak lag. As of yet, the source of the pulse paradigm remains a mystery. This paper does not attempt to explain the origin of the pulse lag, but assumes that it derives from a process common to all bursts and has some proper decay timescale $\Delta t^{\prime}$ in the reference frame of the emitter.

In this paper I will present an interpretation of the luminosity-lag relationship as being due to relativistic motion of the emitting region toward the observer. Thus it is a purely kinematic effect.

\section{Photon Number Luminosity and Cos- mology}

In Norris et al. (2000a) a peak $\gamma$-ray luminosity $L_{p k}$ was defined from the observed peak number flux $F_{p k}$, a luminosity distance $D_{L}$ derived from the observed redshift $z$ and a given cosmology, and a mean emitted photon energy $\bar{\epsilon}$, constant for all bursts:

$$
L_{p k} \equiv 4 \pi \bar{\epsilon} F_{p k} D_{L}^{2} .
$$

However, one does not know the intrinsic energy of the photons emanating from the pulses. Therefore multiplication of all GRB fluxes by an average photon energy $\bar{\epsilon}$, while useful to estimate GRB peak luminosites, obfuscates the underlying relationships in the dynamics of GRBs. In other words, photon number flux $F_{p k}$ is the observed quantity and thus photon number luminosity $N_{p k}$ can be the only calculable luminosity. 
The luminosity distance $D_{L}$ is defined so that redshift of photons is accounted for. The photon number luminosity does not depend on photon energy redshift and thus is given by

$$
N_{p k}=\frac{4 \pi F_{p k} D_{L}^{2}}{(1+z)} .
$$

In Figure 1 is shown the peak number luminosity $N_{p k}$ versus spectral lag $\Delta t$ for six bursts with known redshifts. I fit the data by minimizing $Q^{2} \equiv \Sigma\left(\log \left(N_{p k \text {, model }}\right)-\log \left(N_{p k \text {, data }}\right)\right)^{2}$ where $N_{p k, \text { model }} \equiv A(\Delta t)^{-p}$. The best fit for lags CCF31 0.1 (see Fig. 1) is

$$
N_{p k}=8.6 \times 10^{56} \Delta t^{-0.98} \text { photons sec }{ }^{-1}
$$

with $Q^{2}=1.38$. By comparison, a similar fit of $L_{p k}$ from Eqn (1) versus $\Delta t$ reproduces the Norris et al. (2000a) result $L_{p k} \propto \Delta t^{-1.15}$ with $Q^{2}=1.73$. For CCF31 0.5, $N_{p k} \propto \Delta t^{-0.89}$ with $Q^{2}=0.45$ contrasts with $L_{p k} \propto \Delta t^{-1.03}$ with $Q^{2}=0.79$. With six datapoints, these are small number statistics to be sure. However, the improvement in correlation $N_{p k}$ vs. $\Delta t$ over that of $L_{p k}$ vs. $\Delta t$ via reductions of $Q^{2}$ by $25 \%$ or greater is clear.

\section{Relativistic Kinematics}

The relation $N_{p k} \propto \Delta t^{-0.98}$, where 0.98 is almost 1 , is a strikingly simple statistical property for an ensemble of GRBs to obey. In fact this relationship suggests a simple explanation. I propose that this proportionality is a result of variations in line-of-sight relativistic velocities of GRBs.

For a burst expanding with Lorentz factor $\gamma \equiv$ $1 / \sqrt{1-\beta^{2}}, \beta \equiv v / c$ and at redshift $z$, the observed number luminosity $N_{\text {obs }}$ (photons $\mathrm{sec}^{-1}$ ) varies as

$$
N_{o b s}=\frac{\gamma N^{\prime}}{(1+z)}
$$

where $N^{\prime}$ is the proper isotropic number luminosity for a non-expanding source. This expression is valid for emission that appears isotropic to the observer (i.e. if the emission is in the form of a jet, then the opening angle $\theta_{0}>1 / \gamma$, where $1 / \gamma$ is the relativistic beaming angle). Also, as mentioned previously, if one assumes that the spectral pulse lag is due to some proper decay timescale

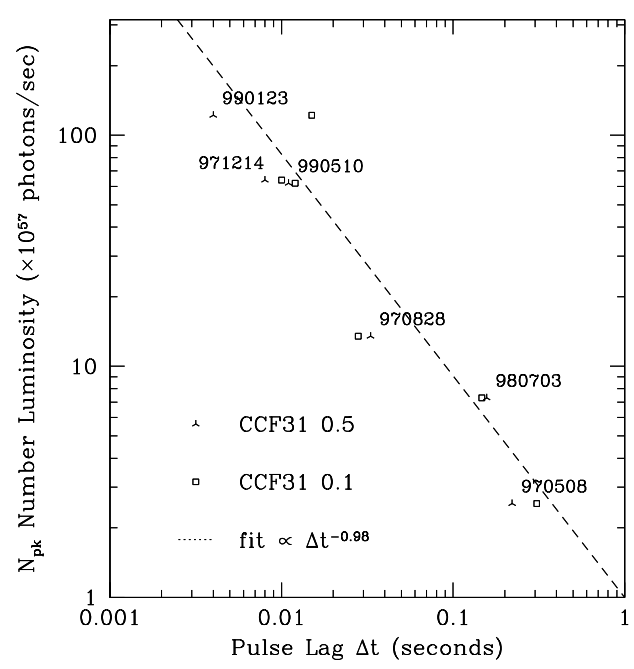

Fig. 1.- Peak photon number luminosity $N_{p k}$ versus spectral pulse lag for six bursts with known redshifts. Spectral cross-correlation function lag between BATSE channels 3 and 1 (CCF31) for regions down to 0.5 and 0.1 of peak intensity were obtained from Norris et al. (2000a). The line of best fit for 0.1 (squares) is $\propto \Delta t^{-0.98}$.

$\Delta t^{\prime}$, possibly due to cooling or deceleration, then we observe a lag

$$
\Delta t_{o b s}=\gamma(1-\beta \cos \theta)(1+z) \Delta t^{\prime} \simeq \frac{(1+z) \Delta t^{\prime}}{2 \gamma}
$$

for $\gamma \gg 1$, where the angular dependence is removed by the strong effect of attenuation of number flux $F$ received from emission moving at inclination angles greater than $1 / \gamma ; F \sim(\gamma(1-$ $\beta \cos \theta)(1+z))^{-3}$. Thus one can focus one's attention only on emitters moving directly toward the observer. Combining these then yields the inverse relationship

$$
N_{o b s} \propto \Delta t_{o b s}^{-1} .
$$

This relationship is redshift independent and thus is valid for luminosity $N_{p k}=(1+z) N_{o b s}$ and spectral lag $\Delta t=\Delta t_{\text {obs }} /(1+z)$ as would be observed local to the burst. Thus we have an explanation of the relation shown in Figure 1. I propose that the variety in the spectral lags and number luminosities of bursts depend solely upon the relativistic 
motion of the emitter along the line of sight. Certainly one expects variation among bursts to cause deviations from this relationship, however the current data suggests this effect to be dominant.

There are two possibilities for the nature of this variation (see Section 6). First, we are observing an intrinsic range of burst expansion speeds, ranging from fast bursts having little or no spectral lag to slower bursts having longer lags. The second possibility is that emitting material is in the form of a jet with some angular dependence on the emitter's Lorentz factor $\gamma(\theta)$ where the Lorentz factor would have a peak at the centroid of the jet $\gamma(\theta=0)=\gamma_{\max }$ and monotonically decrease on some angular scale greater than $1 / \gamma$ for increasing inclination angles $\theta$. In either case we observe only the emission dominated by those emitters moving with trajectories inclined within an angle $1 / \gamma$ from our line-of-sight.

\section{Redshift Estimation}

The above discussion allows the estimation of redshift $z$ from the observed peak number flux $F_{p k}$ and the observed spectral lag $\Delta \tau_{o b s}$. Fenimore \& Ramirez-Ruiz (2000) also estimate redshifts using GRB variability to indicate luminosity. From Eqn (2) one has

$$
N_{p k}=4 \pi F_{p k}(1+z)^{3} D_{A}^{2}
$$

where $D_{A}(z)$ is the angular diameter distance (e.g. Hogg 1999) which is a function of the cosmology and redshift $z$, but does not vary much over the interval $1 \lesssim z \lesssim 3$. For example, here I follow Norris et al. (2000a) and choose $\left(\Omega_{M}, \Omega_{\Lambda}\right)=(0.3,0.7)$, for which $D_{A} \approx 0.4 D_{H}$ to within $10 \%$ over this range of $z$, where $D_{H} \equiv c / H_{0}$ is the Hubble Distance (choose $H_{0}=65 \mathrm{~km} \mathrm{sec}^{-1} \mathrm{Mpc}^{-1}$ ). Also, an observed spectral lag $\Delta t_{o b s}$ varies from the local spectral lag $\Delta t$ by

$$
\Delta t=\frac{\Delta t_{o b s}}{(1+z)} .
$$

One can put these components into the $N_{p k}(\Delta t)$ curve of bursts with known redshifts

$$
N_{p k}=\frac{A}{\Delta t},
$$

where $A=8.6 \times 10^{56}$ photons as taken from the fit in Figure 1. Thus one can estimate a redshift for a given observed peak number flux and spectral lag

$$
z \simeq \sqrt{\frac{A}{4 \pi F_{p k} D_{A}^{2} \Delta t_{o b s}}}-1 \simeq \frac{1.5}{\sqrt{F_{p k} \Delta t_{o b s}}}-1
$$

where flux $F_{p k}$ is measured in photons $\mathrm{cm}^{-2} \mathrm{sec}^{-1}$ and $\Delta t_{o b s}$ is in seconds. It is worth noting that a well determined cosmology gives a well determined $D_{A}(z)$ and thus a refined redshift $z$. Conversely, many independently determined redshifts might give cosmological information. However, the primary source of error in this relation is not the uncertainty in $D_{A}(z)$, but in the deviations of burst luminosities and lags from the relation of Eqn (9) as can be seen in Figure 1.

\section{Observed Peak Pulse Energy}

In order for there to be a lag $\Delta t$ of the arrival of a pulse over an energy range fixed with respect to the observer (e.g. the range between BATSE channels 1 and 3) that varies inversely with Lorentz factor (Eqn 5), the peak energy of the pulse must decay exponentially as

$$
E_{p k} \simeq E_{p k, 0}^{\prime} \gamma \exp \left(-\frac{t \gamma}{\tau_{p k}}\right)
$$

where $E_{p k, 0}^{\prime}$ is the peak energy in the emitter frame, $t$ is observer time, $\gamma$ is the Lorentz factor of the emitter and $\tau_{p k}$ is the proper decay timescale in the emitter frame. Thus we infer an exponential decay law for the pulse peak energy.

It is important to note that this exponential evolution can be very rapid $(\lesssim 10 \mathrm{msec}$ ) (Norris et al. 1996, 2000a) and is distinct from the evolution of the pulse envelope (the so-called FRED) that has been studied by many authors (e.g. Ryde \& Svensson 2000; Liang \& Kargatis 1996; Norris et al. 1996; Fenimore \& Bloom 1995).

One outstanding question in the study of GRB pulses is the apparent invariance among bursts of the peak energy $E_{m x}$ of the $E^{2} N_{E}$ flux spectrum $\left(E_{m x} \sim\right.$ a few $\left.\times 100 \mathrm{keV}\right)$ where $N_{E}$ is number flux per photon energy. One might expect this value to vary with burst luminosity, but it remains roughly constant (to a factor of a few) over a wide variety of (long) GRBs.

If the pulse photon number flux $N_{E}$ (which, for demonstration, we assume to be peaked at $E_{p k}$ ) 
increases as the peak photon energy $E_{p k}$ of Eqn (11) decreases, then there will be a maximum in $E_{p k}^{2} N_{E}$. This may happen as follows: the nascent pulse emitter is extremely hot and compact. As it evolves, it cools ( $E_{p k}$ drops) and it expands or becomes progressively more optically thin, releasing an ever increasing photon rate $N_{E}$. This is analogous to early-time supernova lightcurves (Eastman et al. 1994). In this picture, uncovering the flux dependence $N_{E}(t, \gamma)$ will give insight into why $E_{m x} \approx$ constant. For instance $N_{E} \propto t^{\ln \gamma}=\gamma^{\ln t}$ with Eqn (11) demonstrates this behavior.

\section{Discussion}

In this letter I have argued that the correlation of Norris et al. (2000a) is between number luminosity and spectral lag and I interpret this as being due to the variety, among bursts, of relativistic velocities at which emitting regions move toward the observer. Suppositions as to the physical emission process have been deliberately avoided in order to highlight this purely kinematic effect.

There are two possible scenarios by which this might happen. The first is simply that the variation is due to the variation of expansion Lorentz factor among bursts. From Eqn (5), this implies that the ratio of maximum to minimum spectral lags is the same as the ratio of maximum to minumum Lorentz factors

$$
\frac{\gamma_{\max }}{\gamma_{\min }}=\frac{\Delta t_{\max }}{\Delta t_{\min }} \gtrsim 100
$$

where observed lags are seen to range over roughly two orders of magnitude. Thus if the fastest bursts have $\gamma_{\max } \sim 100$, then the minimum bursts would have $\gamma_{\min } \sim 1$ and thus may be only mildly relativistic.

The second possibility is that GRB ejecta is directed in a jet such that the Lorentz factor $\gamma(\theta)$ has some maximum $\gamma(\theta=0)=\gamma_{\max }$ and monotonically decreases with increasing angle. Thus the function $\gamma(\theta)$ will determine the relative numbers of bursts observed with given spectral lags. Band (1997) has noted that the distribution of bursts is strongly peaked at small lags indicating that the Lorentz factor of emitting ejecta, $\gamma\left(\theta<\theta_{0} / 2\right) \sim \gamma_{\max }$, is basically constant over the jet opening angle $\theta_{0}$, with a fairly narrow edge region $\theta>\theta_{0}$ of decreasing $\gamma$. Thus let us estimate a characteristic opening angle $\theta_{0}$ by assuming that $\gamma=\gamma_{\max }$ is constant for $\theta<\theta_{0} / 2$ and decreases to order unity within a narrow boundary or "edge" region $0<\theta-\theta_{0} / 2<1 / \gamma_{\max }$. Thus the solid angle subtended by "face" bursts (i.e. observed at inclinations $\left.\theta<\theta_{0} / 2\right)$ is $\pi\left(\theta_{0} / 2\right)^{2}$, and that for "edge" bursts is $\pi \theta_{0} / \gamma_{\max }$ and the ratio is:

$$
\frac{\text { "edge" bursts }}{\text { "face" bursts }}=\frac{4}{\gamma_{\max } \theta_{0}} \text {. }
$$

From Fig. 3 of Norris et al. (2000a), one can estimate this ratio, by defining short lags $<0.1 \mathrm{sec}$ as "face" and the longer lags as "edge" bursts, to be $\sim 2 / 5$. Assuming $\gamma_{\max }=100$, then this gives $5^{\circ}<\theta_{0}<10^{\circ}$. With more statistics of the distribution of bursts along the curve of Eqn. (6) one can fit the function $\gamma(\theta)$ much more precisely.

This work was performed under the auspices of the U.S. Department of Energy by University of California Lawrence Livermore National Laboratory under contract W-7405-ENG-48.

\section{REFERENCES}

Band D. L. 1997, ApJ, 486, 928

Daigne F., Mochkovitch R. 1998, MNRAS, 296, 275

Eastman R. G., et al. 1994, ApJ, 430, 300

Fenimore E. E., Bloom J. S. 1995, ApJ, 453, 25

Fenimore E. E., Ramirez-Ruiz E. 2000, submitted ApJ, astro-ph/0004176

Hogg D. W. 1999, astro-ph/9905116

Liang E., Kargatis V. 1996, Nature, 381, 49

Norris J. P., Marani G. F., Bonnell J. T. 2000, ApJ, 534, 248

Norris J. P., Marani G. F., Bonnell J. T. 2000, in AIP Conf. Proc. 526, Gamma-Ray Bursts: Fifth Huntsville Symposiom, ed. R. M. Kippen et al. (New York: AIP), 78

Norris J. P., et al. 1996, ApJ, 459, 393

Panaitescu A., Mészáros, P. 1998, submitted ApJ, astro-ph/9810258

Ryde F., Svensson R. 2000, ApJ, 529, L13

Wu B., Fenimore E. 2000, ApJ, 535, L29

This 2-column preprint was prepared with the AAS LATEX macros v5.0. 